\title{
LARGE-EDDY SIMULATION OF TURBULENT FLOW ABOVE AND WITHIN A FOREST
}

\author{
ROGER H. SHAW ${ }^{1}$ and ULRICH SCHUMANN ${ }^{2}$ \\ ${ }^{1}$ Department of Land, Air and Water Resources, University of California, Davis, CA 95616, U.S.A. \\ ${ }^{2}$ DLR, Institute of Atmospheric Physics W-8031 Oberpfaffenhofen, Germany
}

(Received in final form 12 February, 1992)

\begin{abstract}
A large-eddy simulation has been performed of an atmospheric surface layer in which the lower third of the domain is occupied by a drag layer and heat sources to represent a forest. Subgridscale processes are treated using second-order closure techniques. Lateral boundaries are periodic, while the upper boundary is a frictionless fixed lid. Mean vertical profiles of wind velocity derived from the output are realistic in their shape and response to forest density. Sirnilarly, vertical profiles of Reynolds stress, turbulent kinetic energy and velocity skewness match observations, at least in a qualitative sense. The limited vertical extent of the domain and the artificial upper boundary, however, cause some departures from measured turbulence profiles in real forests. Instantaneous turbulent velocity and scalar fields are presented which show some of the features obtained by tower instrumentation in the field and in wind tunnels, such as the vertical coherence of vertical velocity and the slope of structures revealed by temperature patterns.
\end{abstract}

\section{Introduction}

The air layers within and above a forest, as for any surface composed of relatively tall roughness elements, are linked by turbulent motions at scales large relative to the forest depth that are strongly intermittent in character (Finnigan, 1979; Denmead and Bradley, 1985). This large-scalc intermittency imposes difficulties in the formulation of mathematical models created to simulate exchanges between a forest and the lower atmosphere. The majority of attempts to model turbulent exchange between the atmosphere and tall vegetation, involve first- or higherorder closure schemes based on ensemble-averaged statistics, which are inadequate in representing the intermittent character of the flow. A possible exception to such techniques is the "surface renewal" procedure, which attempts to simulate downbursts injecting the canopy layer with fresh air from aloft ( $\mathrm{Li}$ et al., 1985). It is the existence of large downbursts, which immerse the full depth of the forest, that contradicts local closure and creates the most difficult part of mathematically representing the exchange process.

Further advances in simulation of the interaction of forest and atmosphere must be preceded by improved understanding of the physics of turbulence in the vicinity of the canopy. Until not much more than a decade ago, information of canopy turbulence was limited to spectral and non-spectral statistics with averaging times of a few tens of minutes. Conditional statistical analyses such as quadrant analysis (Finnigan, 1979; Shaw et al., 1983; Baldocchi and Meyers, 1988) strongly implied that the flow was intermittent and, in the canopy layer, dominated by downbursts 
(sweeps) of relatively short duration but which make large contributions to the total vertical transport.

Based on a series of short-interval average profiles of temperature in a pine forest, Denmead and Bradley (1985) depicted the penetration of a gust of relatively cool, dry air into the canopy. Gao et al. (1989) took this a step further by demonstrating the existence of a sharp, sloping frontal boundary between relatively warm, humid air being expelled from the forest and cooler, drier air being swept into the canopy from aloft. Scalar traces at single points showed the microfronts as a series of ramps; scalar values increased with time but this trend was abruptly terminated by a rapid drop before repetition of the pattern. Also revealed was the close relationship between the scalar and vector fields. Gao et al. (1989) and Bergström and Högström (1989) both established the relatively large contribution being made by such coherent structures to the average vertical fluxes of heat and momentum.

A link between the scalar and vector signatures observed by tower instrumentation and large-scale organized motions has yet to be made. Raupach et al. (1989) visualize the creation of transverse vortices resulting from the strong wind speed gradient centered near the top of the canopy but it is not possible to confirm such speculation with existing field data. It is also not known whether such flows relate to the hairpin vortices or double-roller eddies postulated by Townsend (1976), or other vorticular structures that have been observed in smooth-wall boundary layers. Even the most extensive micrometeorological campaigns at forest sites using multiple 3-dimensional anemometry have yielded only a restricted view of the large-scale turbulence. Future field studies might be designed to confirm or reject hypotheses concerning the existence of particular vorticular patterns but it is unlikely that traditional micrometeorological experiments, using tower-mounted instruments, will be able to formalize the link between single-point scalar and flow signatures and the organized motions responsible for them.

The difficulty of obtaining the three-dimensional structure of the flow through observations suggests that numerical simulations could yield valuable insight into the physics of the problem. Large-eddy simulation (LES) explicitly simulates the dominant energetic turbulent scales resolved by the three-dimensional grid array of the computational domain. With the advent of powerful computers, LES has been applied to a wide variety of flow situations, including the atmospheric boundary layer (Deardorff, 1972; Moeng, 1984; Schmidt and Schumann, 1989; Nieuwstadt et al., 1992). LES has the potential for providing answers to fundamental questions concerning the dynamics of vegetated surfaces, as long as computer resources can be provided to resolve the canopy and a substantial atmospheric layer.

Here, we present the results of a pilot study of an LES of the lower atmosphere which includes, in the lowest third of its domain, a canopy layer expressed as vertical distributions of drag and heat sources. The resolution of individual trees is only partially within range because of grid-size limitations; hence, at this initial 
stage, we have chosen to treat the canopy as a porous body of horizontally uniform area density with constant drag coefficient. Further, we have opted to simplify the input of heat by specifying a vertical profile of heat source through the depth of the forest. Thus, we do not solve an energy balance equation, and heat flux density is horizontally uniform. Because of the fine grid spacing needed to resolve the forest canopy, it has not been possible to include an overlying atmospheric layer of a depth typical of the daytime atmospheric boundary layer. However, it is thought that most of the features characteristic of the interaction of the atmospheric surface layer and a rough surface are retained despite the limitation in the vertical extent of the domain.

\section{Method}

\subsection{The equations}

The LES that we have employed is a method to integrate the three-dimensional grid-volume-average equations describing the balances of mass and momentum and the first law of thermodynamics under the Boussinesq approximation. The equations include additional terms for drag by the canopy elements and heat input to the air in contact with the vegetation. The equations are

$$
\begin{aligned}
& \frac{\partial \bar{u}_{j}}{\partial x_{j}}=0, \\
& \frac{\partial \bar{u}_{i}}{\partial t}+\frac{\partial\left(\bar{u}_{j} \bar{u}_{i}\right)}{\partial x_{j}}=-\frac{1}{\rho} \frac{\partial \bar{p}}{\partial x_{i}}+\frac{\partial}{\partial x_{j}}\left(\nu \frac{\partial \bar{u}_{i}}{\partial x_{j}}-\overline{u_{i}^{\prime \prime} u_{j}^{\prime \prime}}\right)+\beta g \bar{T} \delta_{i 3}+F_{i}, \\
& \frac{D \bar{T}}{D t} \equiv \frac{\partial \bar{T}}{\partial t}+\frac{\partial\left(\bar{u}_{j} \bar{T}\right)}{\partial x_{j}}=\frac{\partial}{\partial x_{j}}\left(\mu \frac{\partial \bar{T}}{\partial x_{j}}-\overline{u_{j}^{\prime \prime} T^{\prime \prime}}\right)+S,
\end{aligned}
$$

where $u_{i}$ is velocity in the $x_{i}$-direction, $p$ is pressure, $\rho$ is air density, $\beta=1 / T$ is the volumetric expansion coefficient of the air, $g$ is the gravitational acceleration, $T$ is temperature, $\delta$ is the Kronecker delta, $F_{i}$ is the drag force acting in the $x_{i}$ direction, and $S$ is the rate at which heat is imparted to the air from the vegetation. The ovcrbar signifies an average over a computational grid cell, while the double prime indicates a departure from this average.

The molecular diffusivities $\nu$ and $\mu$ are neglected, other than their implicit inclusion in energy dissipation (see below) and in direct exchanges of heat and momentum at the surface of elements of the canopy. The Coriolis force has been excluded as it has little direct bearing on the scales of mution with which we are concerned.

The momentum and thermodynamic equations include terms for subgrid-scale (SGS) fluxes, which are determined from the resolved fields and the SGS kinetic energy. We have followed the procedure of Schmidt and Schumann (1989), the 
essential parts of which are reproduced here. The subgrid-scale model integrates a closed equation for SGS kinetic energy $\overline{E^{\prime \prime}} \equiv \overline{u_{i}^{\prime \prime}} / 2$ of the form

$\frac{D \overline{E^{\prime \prime}}}{D t}=-\overline{u_{i}^{\prime \prime} u_{j}^{\prime \prime}} \frac{\partial \bar{u}_{i}}{\partial x_{j}}+\beta g \overline{w^{\prime \prime} T^{\prime \prime}}+\frac{\partial}{\partial x_{i}}\left[\frac{5}{3} l c_{3 m} \overline{E^{\prime \prime}} 1 / 2 \frac{\partial \overline{E^{\prime \prime}}}{\partial x_{i}}\right]-c_{\epsilon m} \frac{\overline{E^{3 / 2}}}{l}-2 \frac{\overline{E^{\prime \prime}}}{\tau}$,

where $\tau$ is a time scale for the drag, to be defined in Equation (10). The term in which this time scale appears represents an additional dissipation process due to canopy drag, as discussed below.

The subgrid-scale momentum and heat fluxes and the temperature variance are obtained from a set of algebraically approximated second-order equations. The following derive from the full set of second-order transport equations by assuming local equilibrium, small departures from isotropy, and common closure assumptions:

$$
\begin{aligned}
& 0=-\left(1-c_{G M}\right)^{2 / 3} \overline{E^{\prime \prime}}\left(\frac{\partial \bar{u}_{i}}{\partial x_{j}}+\frac{\partial \bar{u}_{j}}{\partial x_{i}}\right)+\left(1-c_{B m}\right) \\
& \times\left[\beta g\left(\delta_{13} \overline{u_{j}^{\prime \prime} \overline{T^{\prime \prime}}}+\delta_{j 3} \overline{u_{i}^{\prime \prime} \bar{T}^{\prime \prime}}-\frac{2}{3} \delta_{i j} \overline{u_{3}^{\prime \prime} \bar{T}^{\prime \prime}}\right)\right]-c_{R m} \frac{\overline{E^{\prime \prime}}}{l / 2} \overline{A_{i j}^{\prime \prime}}, \\
& 0=-\left(1-c_{G T}\right) \frac{2}{3} \overline{E^{\prime \prime}} \frac{\partial \bar{T}}{\partial x_{i}}+\left(1-c_{B T}\right) \beta g \overline{T^{\prime \prime 2}} \delta_{i 3}-c_{R T} \overline{\frac{E^{\prime \prime 1 / 2}}{l} \overline{u_{i}^{\prime \prime}} \bar{T}^{\prime \prime}}, \\
& 0=-2 \overline{u_{j}^{\prime \prime} \bar{T}^{\prime \prime}} \frac{\partial \bar{T}}{\partial x_{j}}-c_{\epsilon T} \frac{\overline{E^{\prime \prime}}}{l / 2} \overline{T^{\prime \prime 2}} \\
& l
\end{aligned}
$$

where the momentum fluxes and the anisotropic components of the normal stresses are expressed in the form

$$
\overline{A_{i j}^{\prime \prime}}=\overline{u_{i}^{\prime \prime} u_{j}^{\prime \prime}}-\frac{2}{3} \delta_{i j} \overline{E^{\prime \prime}}, \quad i=1,2,3, \quad j=1,2,3 .
$$

In a consideration of scales of motion inside a canopy, Shaw and Seginer (1985) showed that an energy transfer takes place from both the time mean flow and the large-scale turbulence to the wake scale, as small eddies are shed behind canopy elements. It is believed that this mechanism accelerates the dissipation process because small-scale wake motions dissipate rather quickly and contribute little to the turbulent kinetic energy. In an ensemble average canopy model, it is probably important that the transfer between large and small scales be included, as in Wilson (1988). In a large-eddy simulation, the grid-volume-average momentum equation (Equation (2)) explicitly includes the action of canopy dray on the resolved turbulent velocities. The net effect on subgrid-scale turbulence is less obvious. Here, we have chosen to include a term in Equation (4) to represent the removal of SGS kinetic energy by the action of drag, on the assumption that wake motions are of even smaller scale than those making up the bulk of SGS kinetic 
energy. Formally, the term $2 \overline{E^{\prime \prime}} / \tau$ arises from the scalar product $\overline{u_{i}^{\prime \prime}\left(u_{i}^{\prime \prime} / \tau\right)}$ if $\tau$ is weakly correlated with $u_{i}^{\prime \prime}$. Thus, in this simulation, wake-scale motions are assumed to dissipate rapidly and make no contribution to turbulent kinetic energy.

The length scale $l$ is prescribed as a function of height $z$ above the ground surface and of the mesh size $\Delta$ (we chose an equidistant, isotropic grid array), such that

$$
l=\min \left(\Delta, c_{l} z\right) .
$$

In the ensemble-average model of Wilson and Shaw (1977), it was advantageous to restrict the length scale according to the distance from the surface and the density of the vegetation but, because our grid size is relatively small compared with treetop height, the LES subgrid-scale parameterizations are chosen to be independent of the characteristics of the forest.

All coefficients of the SGS model are selected to match those of Schmidt and Schumann (1989), such that $c_{3 m}=0.20, c_{\epsilon m}=0.845, c_{G m}=0.55, c_{B m}=0.55$, $c_{R m}=3.50, c_{G T}=0.50, c_{R T}=0.50, c_{R T}=1.63, c_{\epsilon T}=2.02$, and $c_{l}=c_{e m}$. Most of these coefficients are derived from spectral estimates in the inertial-convective subrange of locally isotropic turbulence, and have proven to be suitable in the large-eddy simulation of a convective atmospheric boundary layer. Comparisons to other LES models (Nieuwstadt et al., 1992) have shown, however, that simpler SGS models with diffusivities computed from $l \bar{E}^{1 / 2}$ give about the same accuracy; see also Schumann (1991).

The drag force is time dependent and is equal to the product of the local foliage density a (a function only of height), a constant drag coefficient $c_{d}$, and the square of the local velocity, such that the force $F_{i}$ in the $x_{i}$-direction is given by

$$
F_{i}=-c_{d} a V \bar{u}_{i}=-\bar{u}_{i} / \tau
$$

where $V$ is scalar speed. The drag coefficient is isotropic and the force directly opposes the local, instantaneous wind vector. In the study described, the drag coefficient is set to 0.15 on the basis of an analysis of micrometeorological data from a deciduous forest (Shaw et al., 1988).

Unlike the momentum sink, the heat source is treated such that it is constant in time and is horizontally homogeneous; thus, we have made no attempt to follow the details of a time-dependent energy budget within the canopy. It is assumed that solar radiation penetrates the canopy and warms the foliage which, in turn, warms the air in contact with it. The heat source is distributed vertically to yield a prescribed average heat flux at $z=h$, the top of the vegetation, and a mean heat flux profile through the canopy, described by a declining exponential function of the downward cumulative leaf area index, in a manner similar to the distribution of net radiation suggested by Brown and Covey (1966). The strength of the heat source, $S$, included in Equation (3) is then the vertical derivative of the upward kinematic vertical heat flux given by 


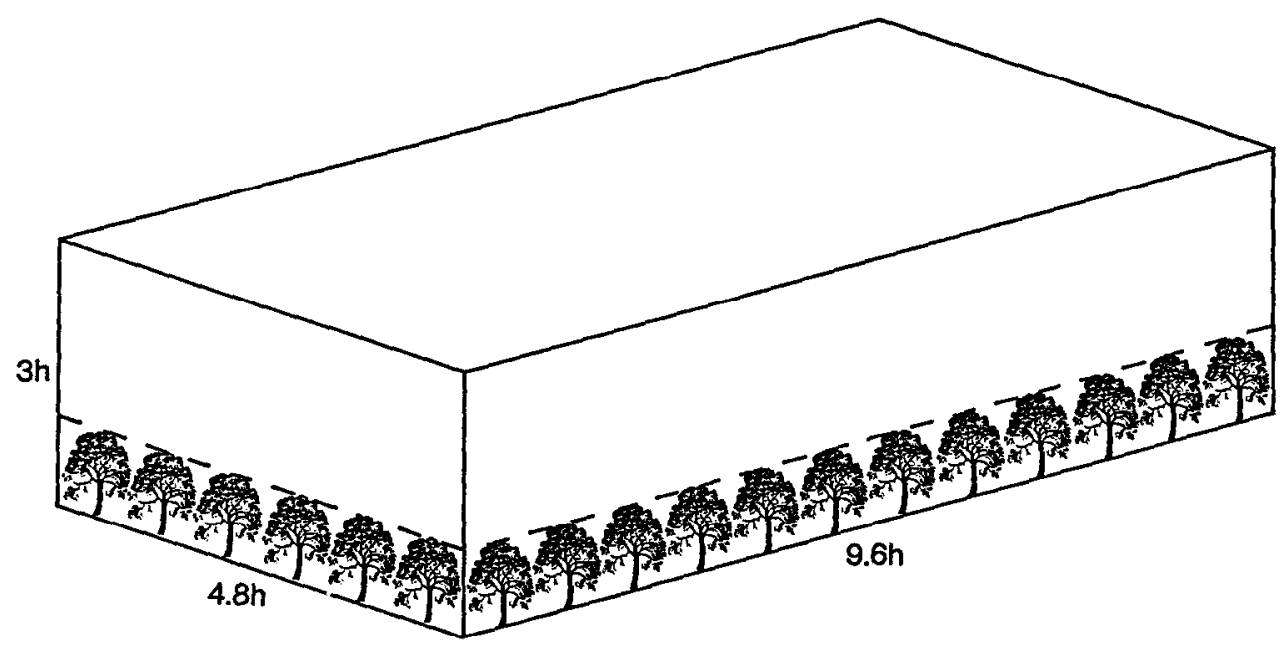

$96 \times 48 \times 30$ grid points

Fig. 1. The computational domain. The mean wind is parallel to the major axis.

$$
Q(z)=Q(h) \exp (-\alpha F), \quad F=\int_{z}^{h} a \mathrm{~d} z
$$

where $F$ is the downward cumulative leaf area index (non-dimensional), and $\alpha$ is an extinction coefficient, which is taken to be 0.6 .

\subsection{NUMERICAL METHOD AND BOUNDARY CONDITIONS}

The integration uses the numerical method MESOSCOP (Schmidt and Schumann, 1989) and is based on an equidistant staggered grid and on finite difference approximations. The momentum and continuity equations are approximated by second-order central differences in space. Time integration is performed using the Adams-Bashforth scheme. The equations for tempcrature and for SGS kinctic energy are approximated by the second-order upwind-scheme of Smolarkiewicz (1984). In the computations, all variables are expressed in non-dimensional form by appropriate scaling using the quantities $U$ (the average longitudinal velocity across a $y, z$ cross-section), $h$ (the total depth of the forest canopy), and $T_{*}$ (a temperature scale defined as $-Q(h) / U)$. Note that $U$ is invariant in the $x$-direction because of continuity.

For this pilot study, the non-dimensional time step $\Delta t U / h$ was chosen to be 0.0125 according to the Courant criterion. The drag force does not limit the time step since $\Delta t<\tau$ in this study. The computations proceeded for 4800 time steps. For a $20 \mathrm{~m}$ tall forest, and a mean velucity $U$ of $2 \mathrm{~m} / \mathrm{s}$, this translates to a simulation of 10 min duration.

The number of grid cells is $96 \times 48 \times 30$ in the $x, y$ and $z$ directions (Figure 1). The forest occupies the lowest 10 grid cells in the vertical and thus the size of the computational domain is $9.6 \times 4.8 \times 3.0$ forest heights. For a $20 \mathrm{~m}$ tall forest, this 


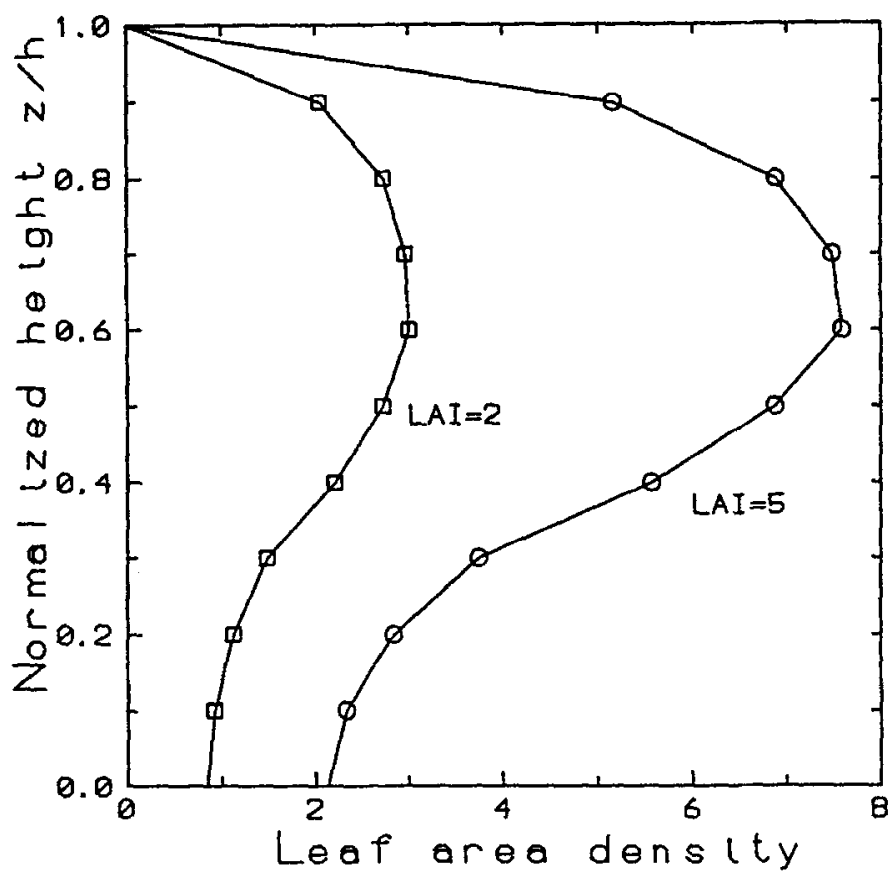

Fig. 2. Vertical profiles of leaf area density (a) for two values of LAI. Leaf area density is normalized by multiplication by the canopy height.

would be $192 \times 96 \times 60 \mathrm{~m}$. The mean flow is aligned with the $x$-direction and a constant mass flow over the cross-section is maintained by adjustment at each time step of a height-independent longitudinal pressure gradient.

The lateral boundaries of the domain are periodic. The upper boundary is treated as a frictionless, rigid lid with zero mass, momentum, heat and SGS kinetic energy flux. This artificial boundary condition may be interpreted as a strong inversion. No-slip conditions are maintained at the lower boundary and stress at the ground is computed from wind velocity at the first grid point above the surface, using the Monin-Obukhov similarity relationships with a prescribed roughness length, $z_{0} / h=0.001$.

The forest canopy was specified as a horizontally uniform leaf area density (area per unit volume of space) with a vertical profile to represent that of a deciduous forest with a relatively open trunk space. The same generic area density profile shape was preserved, while the leaf area index (LAI) was set to values of 5 and 2 during separate computational runs. Plots of the leaf area density profiles are shown in Figure 2.

Three different values of the heat flux were examined, representing convective, more weakly convective, and neutral stability conditions. The three values for the heat flux $\left[Q(h)=0.05,0.005\right.$, and 0 in arbitrary units] result, in order, in $w_{*} / u_{*}$ $=1.6,1.1$, and 0 , where $u_{*}$ is the friction velocity at the top of the forest layer, and $w_{*}$ is the convective velocity scale $\left[w_{*}=(\beta g Q(h) 2 h)^{1 / 3}\right]$. Alternatively, the 
level of instability can be expressed as the ratio of the forest height to the MoninObukhov length. For the three cases, in order, $h / L=-0.80,-0.26$, and 0 . For the neutral case, the heat flux was held at the same value as for the convective run but the volumetric expansion coefficient of the air was set to zero in order to retain temperature as a passive scalar contaminant.

Calculations were performed on a Cray Y-MP. The initial fields were taken as uniform but with small random perturbations added to initiate three-dimensional motions. Previous large-eddy simulations have demonstrated independence of the results, in terms of statistics and typical structures, from such initial conditions. At each time step, the spatially averaged Reynolds stress at the top of the forest was monitored to document convergence to an asymptotic solution. In this state, any remaining trend is much smaller than the turbulent fluctuations. At prescribed intervals, vertical profiles of selected fields were produced by averaging over each horizontal plane. In addition, at the final time step, the turbulent fields of velocity and temperature were obtained as contour and vector plots over various horizontal and vertical slices through the computational array.

\section{Results}

\subsection{Profiles of spatially averaged fields}

Each simulation took about $7000 \mathrm{~s}$ of computer time. This translates to a ratio of about 12 to 1 , computational time to real time (taking $h=20 \mathrm{~m}$ and $U=2 \mathrm{~m} / \mathrm{s}$ ). An asymptotic state was generally achieved after about 1500 time steps; roughly one third of the total simulation period.

Figure 3 shows vertical profiles of instantaneous, horizontally averaged longitudinal velocity for the two values of the LAI. For the velocity profiles, it proved necessary to apply two-point averaging in the vertical to smooth out noise in the profiles induced by the rapid change in the drag force at the canopy top. Smoothing was applied only on the final output and was not part of the computational procedure at each time step. The profiles are of similar shape to observed winds, such as those displayed by Raupach et al. (1991), in showing near-logarithmic shape above and near-exponential decay in the upper half of the forest. The expected response to canopy density is observed. The depletion of the wind inside the forest relative to that above is close to values published by Cionco (1979), who defined a coupling parameter $R_{c}$ equal to the ratio of wind speed at $0.25 \mathrm{~h}$ to that at $1.4 \mathrm{~h}$. For the profiles of Figure 3 , the coupling ratios are approximately 0.16 for $\mathrm{LAI}=5$ and 0.26 for $\mathrm{LAI}=2$. As comparison, Cionco lists coupling ratios for deciduous and coniferous forests ranging from 0.12 to 0.19 . Dense tropical forests had lower coupling ratios, while a deciduous forest in winter is quoted as having a coupling ratio of 0.28 , not very different from that our depleted forest of $\mathrm{LAI}=2$.

Both profiles in Figure 3 show a reversal of the velocity gradient in the lower 


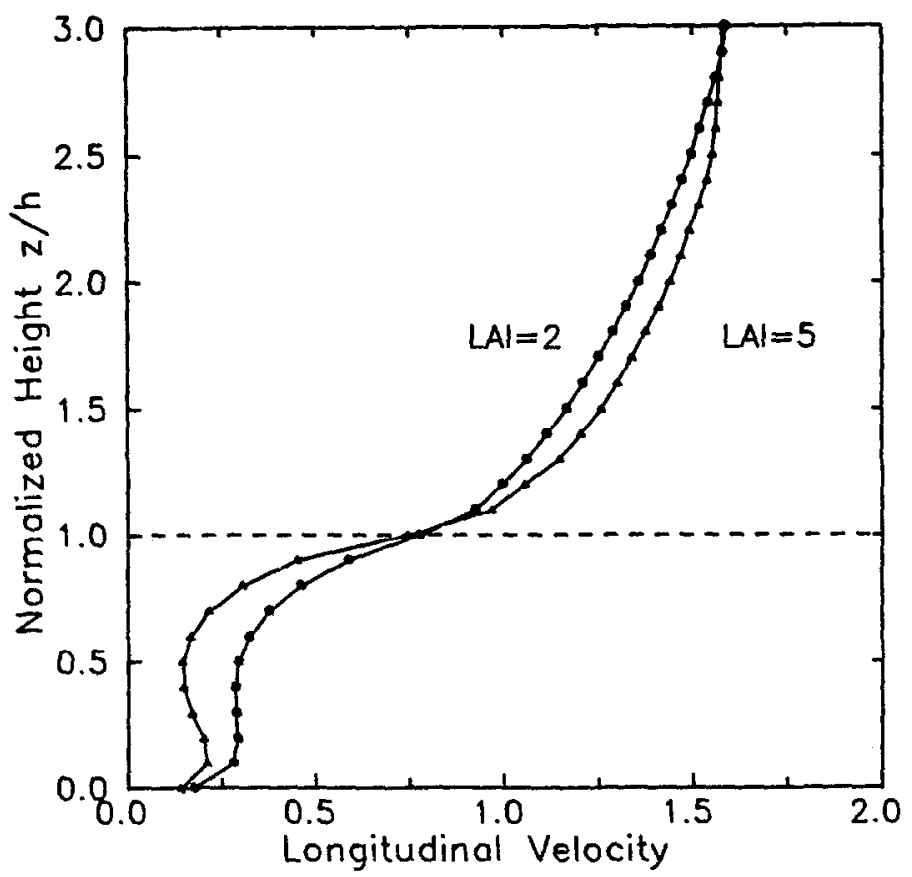

Fig. 3. Spatial mean longitudinal velocity profiles for two values of LAI under weakly unstable conditions. Velocity is normalized by the vertically averaged longitudinal velocity.

half of the canopy but the feature is more pronounced in the case of the denser forest. Such profiles can be indicative of counter-gradient momentum flux, and are a consequence of the frequent flushing of the full depth of the canopy by large eddies penetrating from above, and the relatively small leaf density in the lower part of the forest.

The Reynolds stress decays rapidly inside the forest as demanded by the strong drag forces, but also decays above the canopy in near-linear fashion, as required for steady state, to zero at the top of the domain, as shown in Figure 4, for a case with weak convection and a LAI of 5 . The decrease above the forest is forced by the existence of the frictionless upper boundary and is a departure from a real atmosphere, with a deep mixed layer, in which the first few tens of meters above the forest appear as a near-constant stress layer.

Also shown in Figure 4 is the SGS contribution to the Reynolds stress, indicating that it is generally a very small component of the total momentum flux. Under the more strongly convective situation, the Reynolds stress profile shows slightly deeper penetration of momentum into the forest, while under neutral conditions, there is slightly weaker penetration. For all three cases with $\mathrm{LAI}=5$, the Reynolds stress changes sign in the lower half of the canopy to become small and positive (negative in Figure 4 since the Reynolds stress is normalized by its value at treetop height), representative of an upward flux of momentum. Examination of the 


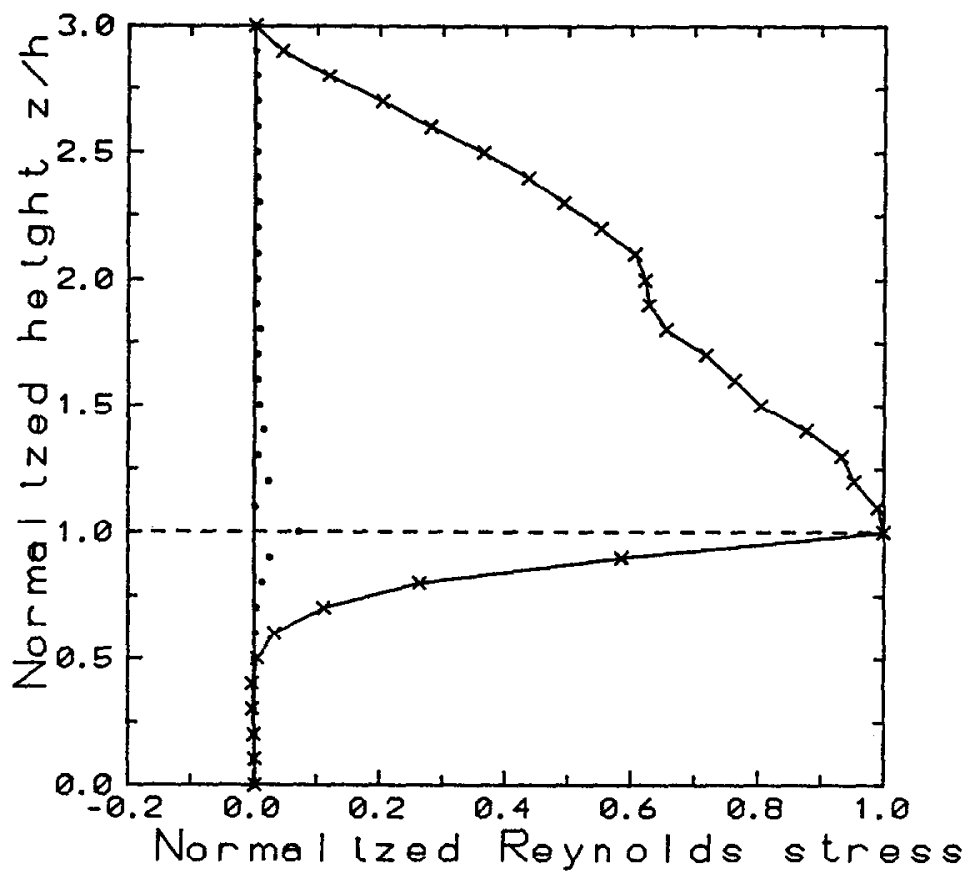

Fig. 4. Vertical profile of the spatial mean Reynolds stress for a LAI of 5 and weakly unstable conditions, and normalized to its value at the top of the canopy. The solid line is the sum of resolved and subgrid-scale components of the Reynolds stress. The dots are the SGS component.

momentum equation reveals that this reversal can only be a consequence of the streamwise pressure gradient.

Figure 5 compares normalized Reynolds stresses for two LAIs, 5 and 2, using the same runs as for the wind profiles in Figure 3. A more extensive penetration into the thinner canopy is expected and is reproduced in the computed flows. Note that for LAI $=2$ the Reynolds stress does not change sign, indicating continued downward flux of momentum, while the velocity gradient reverses sign to a small degree, a definite indication of counter-gradient flux.

The correlation between longitudinal and vertical velocities (not shown) is strongly negative in the upper portion of the forest, achieving values of about -0.6 , and closely matching observations made in a deciduous forest (Shaw et al., 1988).

Other statistical fields examined include vertical profiles of turbulent kinetic energy as shown in Figure 6 for LAI $=5$ and weak instability. Maximum turbulent energy occurs near treetop height and decays rapidly inside the forest. It also decreases to about one third its maximum value at the top of the domain. This decrease above the forest is counter to observations from the field, in which turbulent kinetic energy tends to remain fairly constant or even increase somewhat above the canopy (for example, see Shaw et al., 1988). The decrease shown by the simulated wind field occurs because the fixed upper lid imposes decreases on 


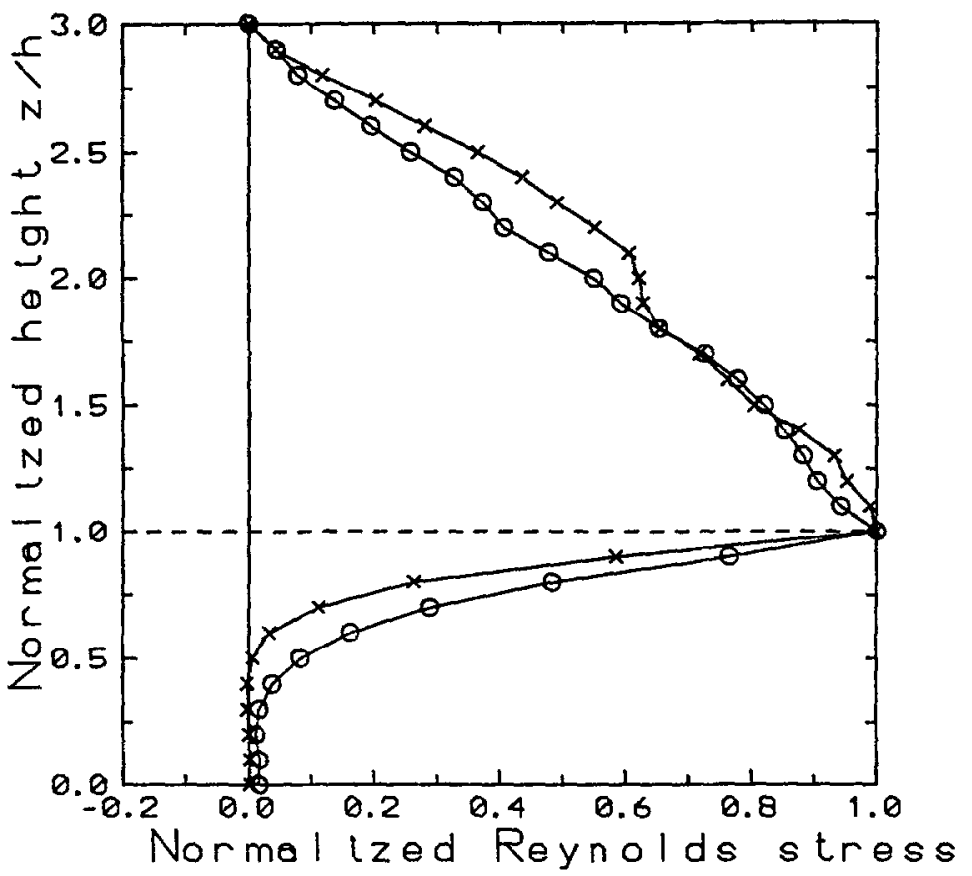

Fig. 5. Vertical profiles of Reynolds stress for LAIs of 2 (open circles) and 5 (crosses), for weakly unstable conditions in both cases.

the momentum and heat fluxes, both of which are components of production terms in the turbulent kinetic energy equation.

The SGS component of the turbulent kinetic energy is larger in a relative sense than it is for Reynolds stress (Figure 4) but is still only about $10 \%$ of the total. From the relatively small contributions by subgrid processes to the Reynolds stress and the turbulent kinetic energy, we conclude that the turbulent flow approaches a locally isotropic state at small scales and that our grid dimensions, with sides equal to one tenth of the forest height, are appropriate for this problem.

The longitudinal and vertical velocities are skewed (Figure 7) in much the same way as found in the field. Inside the forest, vertical velocity is negatively skewed, while longitudinal velocity skewness is positive. Above the forest, these skewnesses are reversed. The sign of the third-order moment of vertical velocity fluctuation indicates turbulent energy transport away from the top of the canopy, where shear production of energy is a maximum, into the lower part of the forest and into the upper part of the mixed layer where dissipation exceeds production of turbulent kinetic energy. The longitudinal velocity skewness reveals strong downstream bursts inside the forest layer and strongly retarded events in the mixed layer above.

Raupach et al. (1991) show velocity skewness profiles from several real and wind tunnel artificial canopies. Our simulations are in qualitative agreement but observations tend to reveal skewnesses of somewhat larger magnitude inside the 


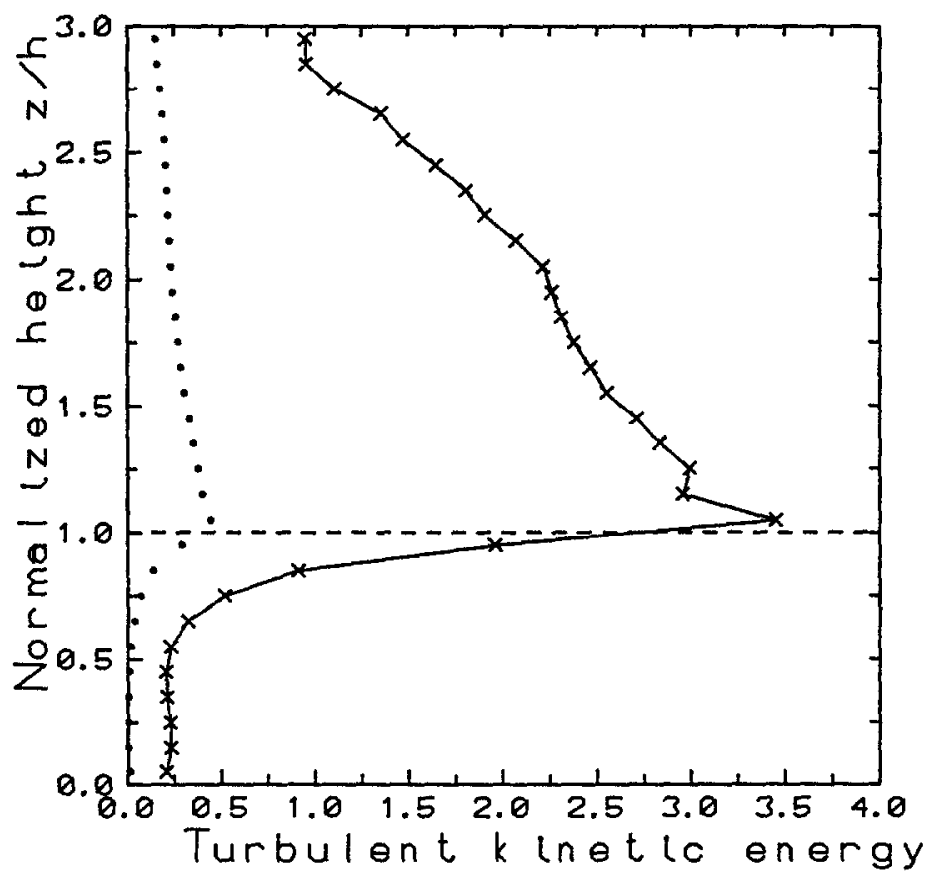

Fig. 6. Vertical profile of turbulent kinetic energy for a LAI of 5 and weakly unstable conditions. Kinetic energy is normalized by the square of the vertically averaged longitudinal velocity. The solid line is the sum of resolved and subgrid-scale components of the turbulent kinetic energy. The dots are the SGS component.

canopy than shown by the simulations. The reversal of both $u$ and $w$ skewnesses above the simulated forest is also seen in field data but, in the field, the change is not so rapid. It is probable that the limited vertical depth of the simulation and the presence of the rigid upper boundary cause the more rapid transition in the sign of the triple moments near the top of the forest.

Overall, statistical properties of the flow fields generated by the LES are reasonable approximations to micrometeorological observations from forest sites, retaining the essential features of turbulence statistics under field conditions.

\subsection{INSTANTANEOUS FIELDS}

An example of an instantaneous field is presented in Figures 8 and 9 of the resolved-scale velocities created by the LES for LAI $=5$ and neutral stability. The figures show contours of longitudinal and vertical velocity for a selected slice through the domain in the $x, z$ plane for the final time step during one of the simulations. Positive and negative values are shown by solid and dashed lines, respectively. Figure 10 shows the same data in vector form. To improve clarity, the vertical dimension in each diagram is enlarged by a factor of 1.6 relative to the horizontal dimension.

The vertical shear of the streamwise velocity (Figure 8) varies down the length 


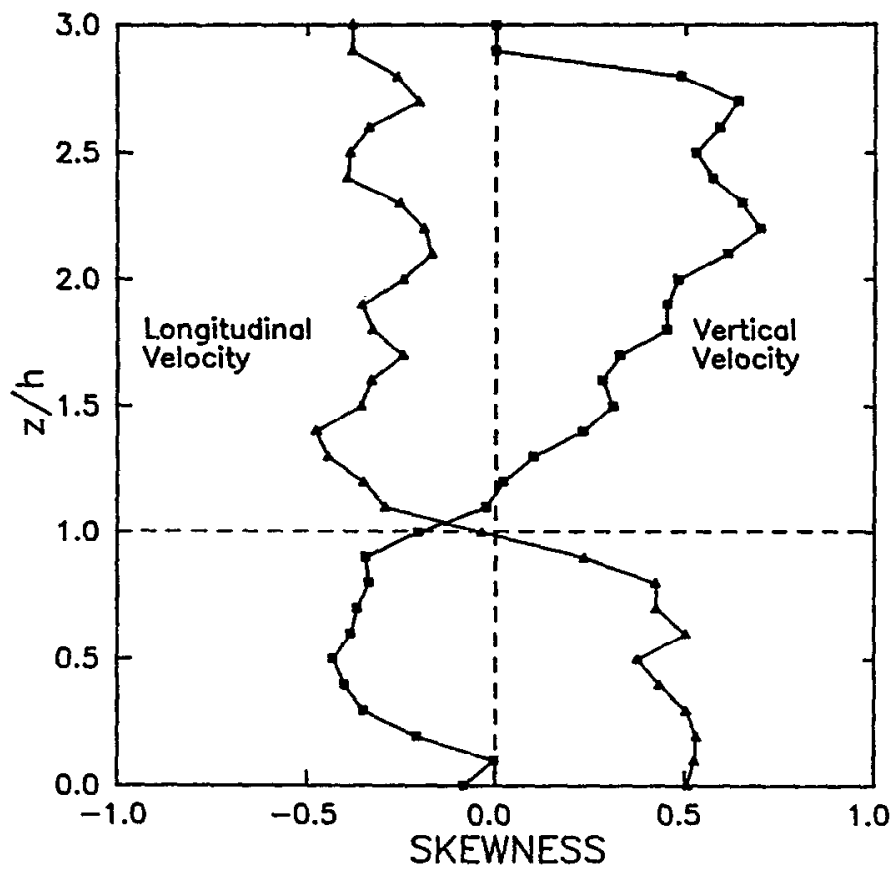

Fig. 7. Vertical profiles of longitudinal and vertical velocity skewness for a forest with LAI of 5 and weakly unstable conditions.

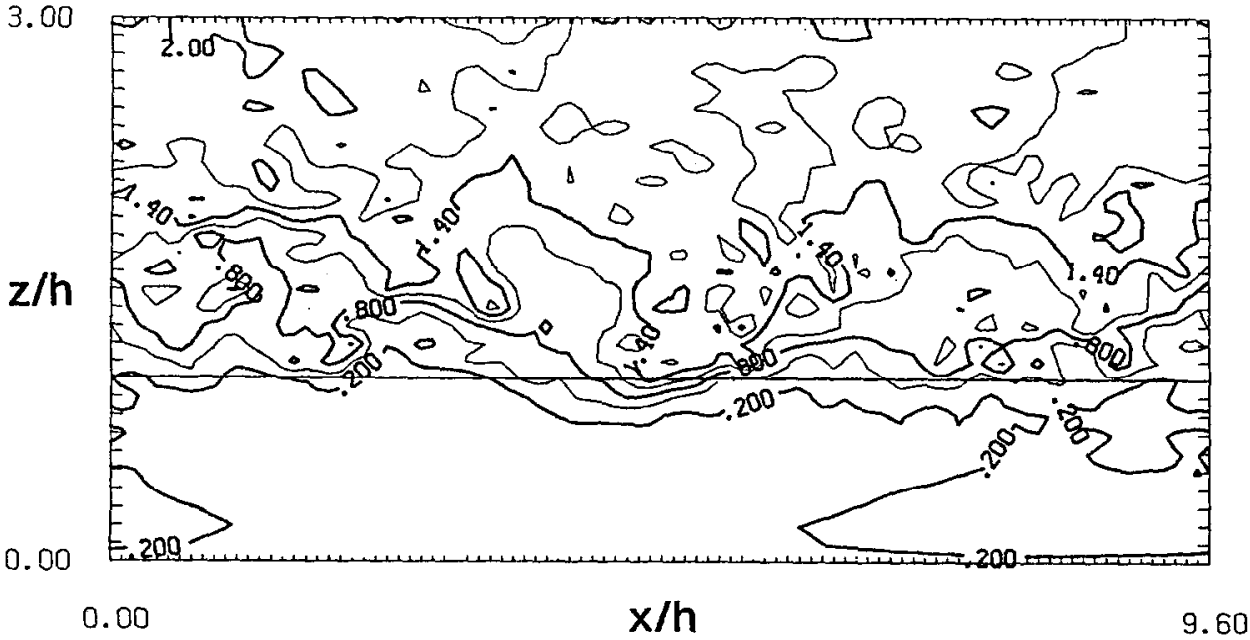

Fig. 8. Instantaneous longitudinal velocity field over an $x, z$ slice midway through the array $(y / h=$ 2.4), for a LAI of 5 and neutral stability. Contour interval is 0.3 in units of velocity normalized by the vertically averaged longitudinal velocity. Spatial scales are normalized by the height of the canopy $h$, which is indicated by the horizontal line. 


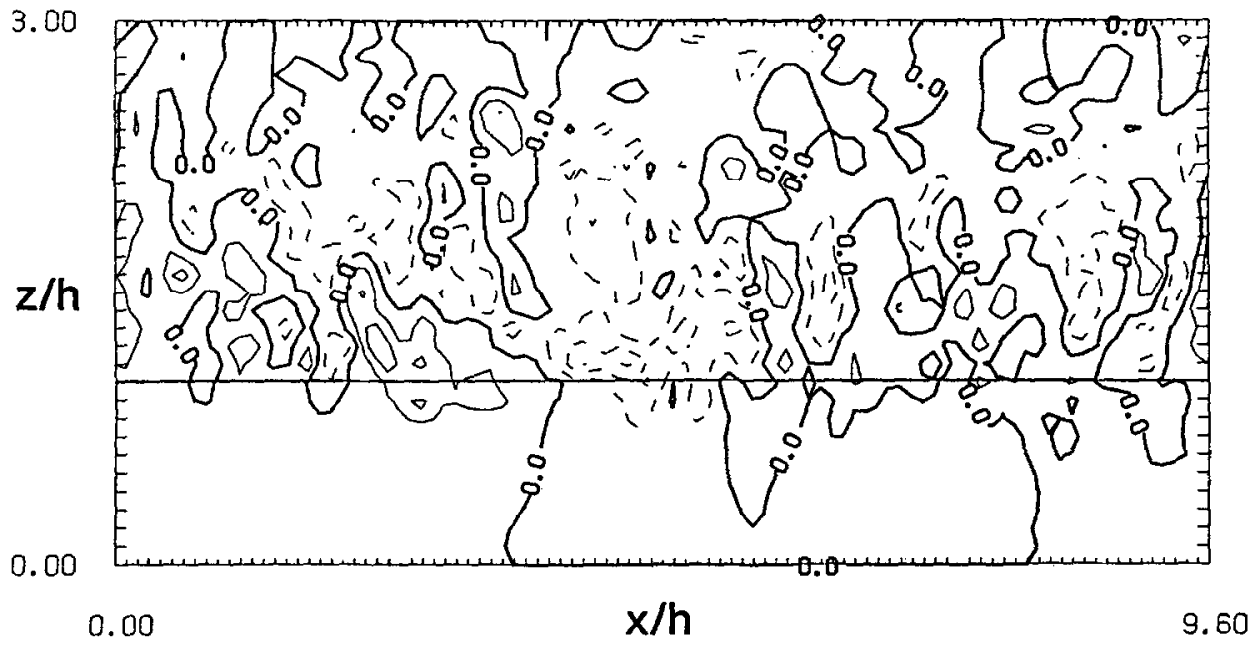

Fig. 9. Same as Figure 8 but for vertical velocity. Contour interval is 0.2 . Solid lines indicate positive values; broken lines indicate negative values.

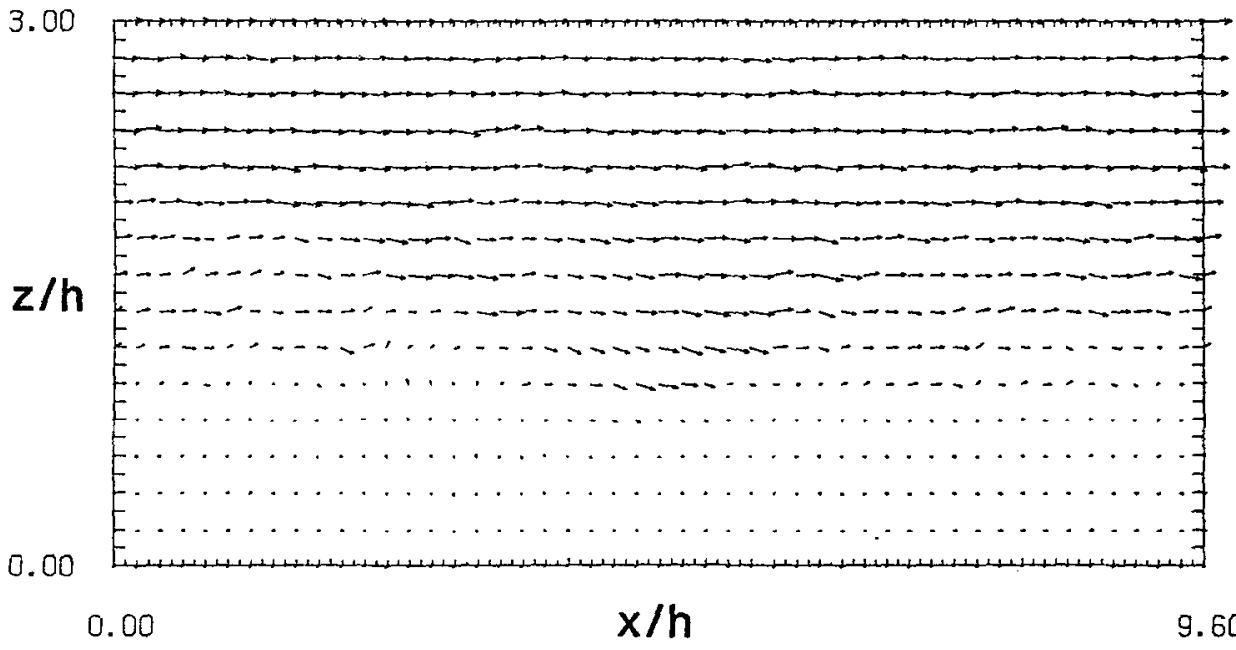

Fig. 10. Streamwise and vertical velocities as in Figures 8 and 9 plotted in vector form. Maximum vector 2.1 in units of the vertically averaged longitudinal velocity.

of the domain and is greatest midway between the upwind and downwind edges in the upper reaches of the forest, where the 1.4 nondimensional velocity contour dips to treetop height. In this same region, shear in the lowest two thirds of the canopy and above the forest is weak. Upstream and downstream of this region, streannwise velocity sliear is more diffuse. Coincident with the zone of high shear, the contour plot of vertical velocity (Figure 9) reveals an area of downdraft that is vertically coherent through most of the depth of the domain, and which has a size in the streamwise direction of about 1.5h. Although not shown here, the cross-stream dimension of this downdraft has been found to be of similar magni- 


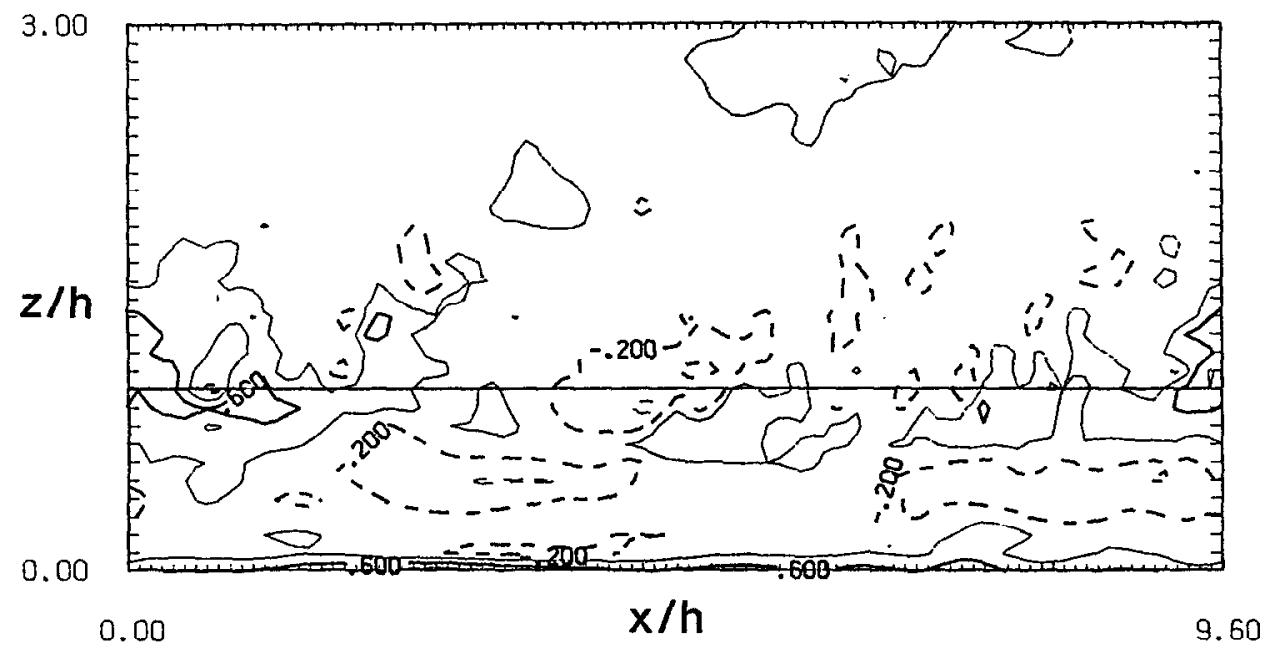

Fig. 11. Same as Figure 8 but for temperature fluctuation (temperature treated as a passive scalar). Contour interval is 0.4 in units of the kinematic heat flux at treetop height divided by the vertically avcraged longitudinal velocity. Solid lines indicate positive values; broken lines indicate negative values, relative to the initial volume-mean temperature.

tude. Clearly, the downdraft and the sharp streamwise velocity gradient in the upper canopy are linked. Areas of the diagram occupied predominantly by updrafts relate well to regions of low velocity near the top of the forest and to weak shear. A smaller scale downdraft located about 2 forest heights from the upwind edge is also accompanied by increased shear near the top of the canopy, although not to the extent of the case already described.

The passive scalar field of temperature (the buoyancy parameter is set to zero for this neutral case) corresponding to the turbulent flow field of the previous diagrams is shown in Figure 11. There is a clear relationship between the scalar and the vector fields with cooler air penetrating the forest from above in the central downdraft, and warmer air leaving the upper canopy in areas of updraft at the left and right sides of the diagram. A feature apparent in the field of temperature perturbations is the inclination in the downstream direction of masses of air cooler or warmer than average. This is most distinctly demonstrated in the below-normal temperatures in the center of the diagram, and in the warmer air leaving the forest upwind of the center. The inclination in the scalar patterns is characteristic of convective plumes in shear flow but, in this case, is not driven by convection. Simulations performed with convective conditions demonstrate a similar tilt to the turbulence structures.

Unlike the case for temperature, visual inspection of Figure 9 fails to reveal any tilt in the pattern of vertical velocity. This difference between the scalar and vector fields is in qualitative agreement with lagged cross-correlations of observations in rough-wall boundary layers (Raupach et al., 1991), that is, temperature exhibits 


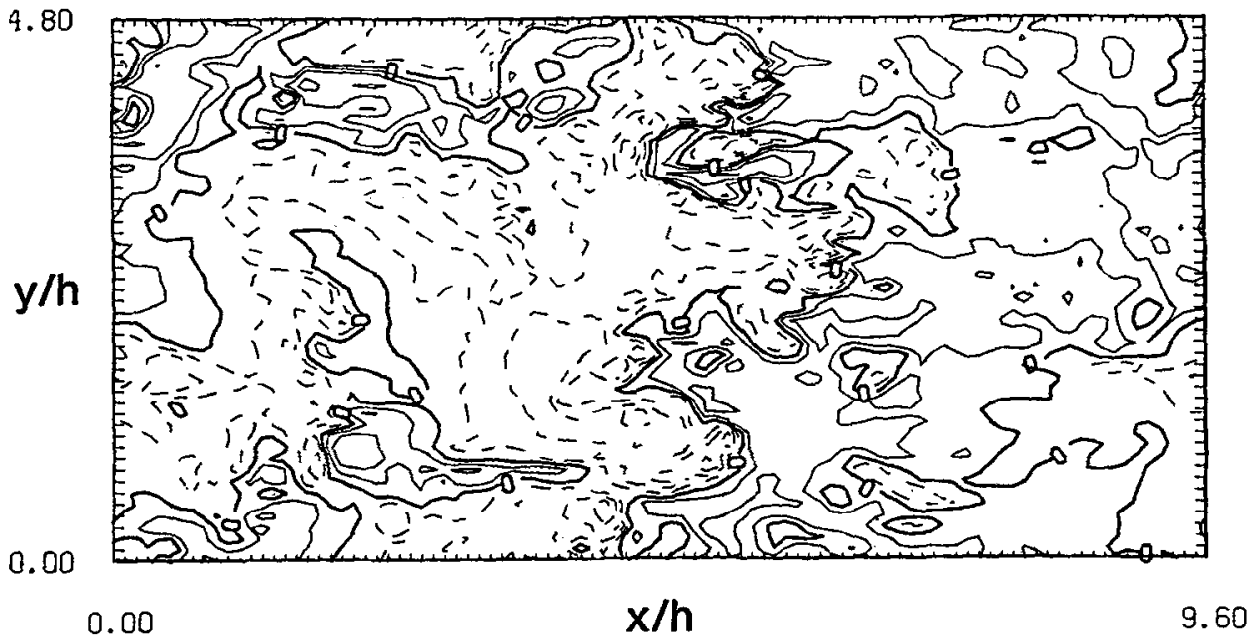

Fig. 12. Horizontal slice through the temperature pattern at treetop height for a LAI of 5 and weakly unstable conditions. Contour interval is 0.02 in units of the kinematic heat flux at treetop height divided by the vertically averaged longitudinal velocity.

increasing lag with decreasing height, while vertical velocity is more or less in phase at all observation levels.

The final diagram presents an example of a horizontal slice through the temperature pattern at treetop height, for $\mathrm{LAI}=5$ and weak convection (Figure 12). Note the large range of scales comprising the turbulence field. Also note that the largest scales, and what are probably the dominant eddies, are large in comparison to the canopy depth. The temperature patterns are strongly correlated with the vertical velocity field (not shown). A positive correlation is required to provide positive mean heat flux at this level.

\section{Summary and Conclusions}

The MESOSCOP numerical method has been modified to perform a large-eddy simulation of the atmospheric surface layer, resolving the air flow through a forest in the lowest one third of the vertical dimension. Sub-grid scale fluxes are approximated in terms of the resolved fields using second-order closure principles. Vertical profiles of mean flow statistics contain the main characteristics of experimental data specific to tall canopies. Examples of the instantaneous fields are presented. Features of the computed turbulence patterns match those found in the atmosphere at forest sites.

While the simulations represent a pilot study, they illustrate the potential for future investigations of canopy turbulence using a new computational tool. Such studies may reveal the structure of the turbulent flow and can be used to deduce simpler turbulence models. However, fine resolution inside the canopy can be gained only if one limits the study to a rather small section of the atmospheric 
boundary layer. Each of the simulations took about $7000 \mathrm{~s}$ on a Cray Y-MP. Perhaps a doubling of the size of the domain of the LES is feasible, or fewer than 10 grid points might be sufficient to resolve the canopy in the vertical, leaving more grid points to resolve a larger part of the atmospheric boundary layer. A future possibility is to examine properties of spatially inhomogeneous canopies.

\section{Acknowledgement}

We thank Dr. Jutta Graf for her help in performing the computations, and students Jie Qiu and Xijie Zhang for help in producing some of the figures.

\section{References}

Baldocchi, D. D. and Meyers, T. P.: 1988, 'Turbulence Structure in a Deciduous Forest', Boundary-Layer Meteorol. 43, 345-364.

Bergström, H. and Högström, U.: 1989, 'Turbulent Exchange above a Pine Forest II. Organized Structures', Boundary-Layer Meteorol. 49, 231-263.

Brown, K. W. and Covey, W.: 1966, 'The Energy-Budget Evaluation of the Micro-meteorological Transfer Process within a Cornfield', Agric. Meteorol. 3, 73-96.

Cionco, R. M.: 1979, 'A Summary of an Analysis of Canopy Flow Coupling for a Variety of Canopy Types', pp. 105-106, preprint volume, 14th Conference of the American Meteorological Society on Agriculture and Forest Meteorology, Minneapolis, Minnesota, April 1979.

Deardorff, J. W.: 1972, 'Numerical Investigation of Neutral and Unstable Planetary Boundary Layers', J. Atmos. Sci. 29, 91-115.

Denmead, O. T. and Bradley, E. F.: 1985, 'Flux-Gradient Relationships in a Forest Canopy', in B. A. Hutchison and B. B. Hicks (eds.), The Forest-Atmosphere Interaction, D. Reidel, Dordrecht, pp. 421-442.

Finnigan, J. J.: 1979, 'Turbulence in Waving Wheat II. Structure of Momentum Transfer', Boundary-Layer Meteorol. 16, 213-236.

Gao, W., Shaw, R. H., and Paw U, K. T.: 1989, 'Observation of Organized Structure in Turbulent Flow within and above a Forest Canopy', Boundary-Layer Meteorol. 47, 349-377.

Li, Z. J., Miller, D. R., and Lin, J. D.: 1985, 'A First-Order Closure Scheme to Describe CounterGradient Momentum Transport in Plant Canopies', Buundary-Layer Meteorol. 33, 77-83.

Moeng, C.-H.: 1984, 'A Large-Eddy-Simulation for the Study of Planetary Boundary-Layer Turbulence', J. Atmos. Sci. 41, 2052-2062.

Nieuwstadt, F. T. M., Mason, P. J., Moeng, C.-H., and Schumann, U.: 1992, 'Large-Eddy Simulation of the Convective Boundary Layer: A Comparison of Four Computer Codes', Turbulent Shear Flows 8 (F. Durst et al., eds.), Springer-Verlag, Berlin, in press.

Raupach, M. R., Antonia, R. A., and Rajagopalan, S.: 1991, 'Rough-Wall Turbulent Boundary Layers', Applied Mechanics Reviews 44, 1-25.

Raupach, M. R., Finnigan, J. J., and Brunet, Y.: 1989, 'Coherent Eddies in Vegetation Canopies', Proc. Fourth Australasian Conf. on Heat and Mass Transfer, Christchurch, New Zealand, 9-12 May, pp. 75-90.

Schmidt, H. and Schumann, U.: 1989, 'Coherent Structure of the Convective Boundary Layer Derived from Large-Eddy Simulations', J. Fluid Mech. 200, 511-562.

Schumann, U.: 1991, 'Subgrid Length-Scales for Large-Eddy Simulation of Stratified Turbulence', Theor. Comput. Fluid Dyn. 2, 279-290.

Shaw, R. H., den Hartog, G., and Neumann, H. H.: 1988, 'Influence of Foliar Density and Thermal Stability on Profiles of Reynolds Stress and Turbulence Intensity in a Deciduous Forest', Boundary-Layer Meteorol. 45, 391-409.

Shaw, R. H. and Seginer, I.: 1985, 'The Dissipation of Turbulence in Plant Canopies', pp. 200- 
203, preprint volume, 7th Symposium of the American Meteorological Society on Turbulence and Diffusion, Boulder, Colorado, November 1985.

Shaw, R. H., Tavangar, J., and Ward, D. P.: 1983, 'Structure of the Reynolds Stress in a Canopy Layer', J. Clim. Appl. Meteorol. 22, 1922-1931.

Smolarkiewicz, P. K.: 1984, 'A Fully Multidimensional Positive Definite Advection Transport Algorithm with Small Implicit Diffusion', J. Comput. Phys. 54, 325-362.

Townsend, A. A.: 1976, 'The Structure of Turbulent Shear Flow', Cambridge University Press, Cambridge, $429 \mathrm{pp}$.

Wilson, N. R. and Shaw, R. H.: 1977, 'A Higher Order Closure Model for Canopy Flow', J. Appl. Meteorol. 16, 1197-1205.

Wilson, J. D.: 1988, 'A Second-Order Closure Model for Flow Through Vegetation', Boundary-Layer Meteorol. 42, 371-392. 